\title{
Index Finger Reflection and Smoking
}

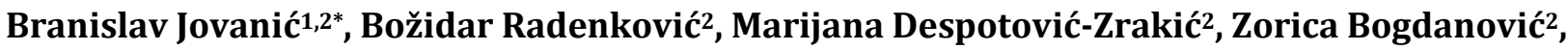 \\ Dušan Barać ${ }^{2}$
}

\author{
${ }^{1}$ Laboratory for Multidisciplinary Research, Institute of Physics, University of Belgrade, Belgrade, Serbia \\ ${ }^{2}$ Laboratory for Simulation, Faculty of Organizational Sciences, University of Belgrade, Belgrade, Serbia \\ Email: ^branislav.jovanic@ipb.ac.rs
}

How to cite this paper: Jovanić, B., Radenković, B., Despotović-Zrakić, M., Bogdanović, Z. and Barać, D. (2020) Index Finger Reflection and Smoking. Health, 12, 1371-1378. https://doi.org/10.4236/health.2020.1210098

Received: September 17, 2020

Accepted: October 24, 2020

Published: October 27, 2020

Copyright (C) 2020 by author(s) and Scientific Research Publishing Inc. This work is licensed under the Creative Commons Attribution International License (CC BY 4.0).

http://creativecommons.org/licenses/by/4.0/

\begin{abstract}
The reflectance of the index finger on $680 \mathrm{~nm}$ was measured. The experimental results show that the intensity of the reflectance for the index finger is related to age and the smoking circumstance. It was shown that reflectance for the index finger nonlinearly increases and reaches the maximum at about 31 - 32 age for nonsmoker age. Index finger reflectance between smoker and nonsmoker was compared. It was observed that smokers' index finger reflectance is significantly greater compared with the index finger reflectance for nonsmokers.
\end{abstract}

\section{Keywords}

Index Finger, Reflectance, Human Skin, Biological Object

\section{Introduction}

Skin is the boundary between the inside body and the environment. The optical characteristics of the skin are strongly determined by the physiological and pathological conditions of the body. So, if different environmental factors cause changes in physiology and pathology of the body, one of the possible consequences can be a change of the skin's optical characteristics. On the other hand, all biological responses to light are the result of chemical and/or physical changes induced in biological systems when they absorbed light energy. The absorption of the light by biological molecules is strongly connected with the dissipation of this energy via heat and light emission and chemistry. Therefore, as reflectance is one of the main optical characteristics of the skin, measuring the reflectance of the skin can give useful information about the physiological and pathological conditions of the body or skin.

The optical properties of human skin have been of interest to researchers for 
some time. The interest was mainly based on a need to study a variety of different applications. In literature, one can find examples of researching reflectance on the skin on different parts of the human body, such as wrist skin [1] finger [2], inside of the forearm [3]. The skin reflection was intensively considered in order to help in the design and evaluation of the reflectance oxygen sensor [4].

The primary goal of this study is to investigate the differences in index finger skin reflection characteristics between smokers and nonsmokers, and also to find out of the obtained differences moderated by age. The results should give new insights into investigating the physiological and pathological condition of human body by considering the optical properties of the index finger skin.

\section{Experimental}

The object of measurement was the index finger, from the left human hand. Before measuring, the finger was washed in water and dried in hot air. The respective measurements of this work have been done in the following way: the fingertip has been lighted by LEDs of $680 \mathrm{~nm} \pm 20 \mathrm{~nm}$. The diode was turned on for 1 minute. During fingertip lighting, the intensity of the reflected light was measured with a photo detector-photodiode. The photodiode worked under the circuit regime, which enabled its stable operation in the surrounding temperature changes. The measurement geometry was always equal, unchanged throughout all measurements since the LED and the photodiode were fixed in the specially constructed hemisphere so that the angle between the incident and reflective radiation was 900 . The signals from the photodiode were reinforced $(10,000$ times) and displayed. All results were normalized on the reflectance of standard $\mathrm{MgO}$ powder. Experimental setup is shown in Figure 1.

In the experiment, we have used convenience sampling method. There were more than 2000 participants in the experiment, all between 25 and 60 years old.

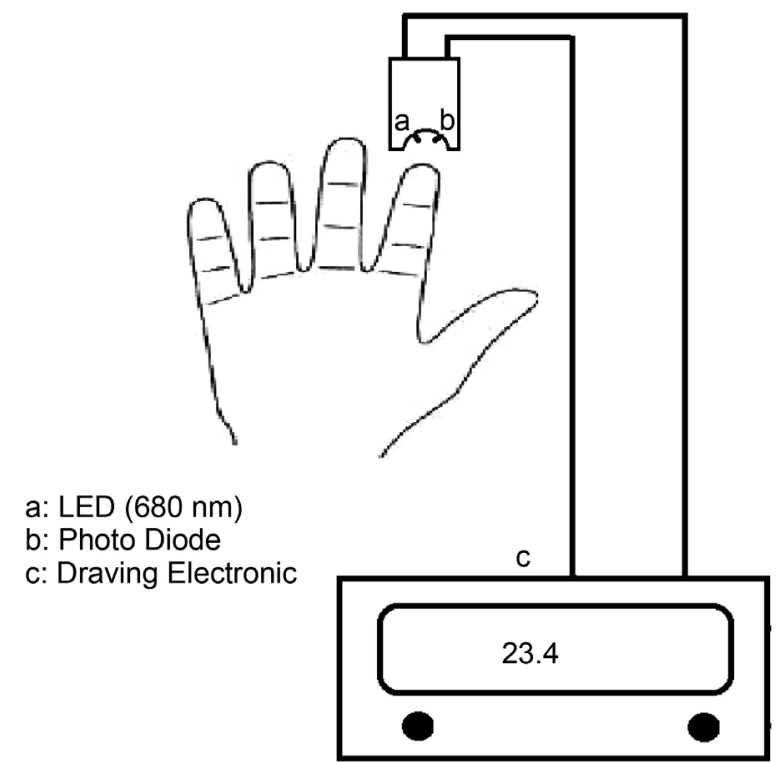

Figure 1. Experimental setup. 
The number of males and females was approximately the same (around $50 \%$ of each), as well as the number of smokers and nonsmokers in each age group. The number of measurements for each investigated group varied between 187 and 200 measurements. Experiments were performed in a long period, for about 21 years, in the Laboratory for Multidisciplinary Research in the Institute of Physics and Cardiovascular Diseases, Emergency Clinical Center in Belgrade. In the mentioned period, we have made a huge database. The sample size completely satisfies the criterion of the limit condition, as for any physical occurrences which depend on one stochastic argument [5]. Therefore relative errors were less than $\pm 2 \%$.

\section{Theory}

There are a lot of examples in literature that prove that a drastic change in physiological and pathological conditions in skin or tissues causes changes in optical spectroscopic attributes. Sunburn skin of humans and animals show a difference in the intensity of fluorescence upon exposure to the sun [6]. Human skin shows a strong dependence of fluorescence intensity upon the activity of propionic bacteria acne [7]. The emission rate of a fingertip is strongly related with the age, the tumor presence, and lipid concentration in the serum [8]. A drastic change in steady-state fluorescence and excitation spectra from human native and malignant breast tissues was observed [9].

The mentioned discussion leads to the conclusion that smoking, as an unfavorable factor, can cause a change in physiological and pathological conditions in the human body and the direct consequence can be a change of skin reflectance. Therefore, measuring the reflectance of the skin can show the physiological and pathological conditions of a human body. The excited wavelength of 680 $\mathrm{nm} \pm 0.4 \mathrm{~nm}$ and emission of $680 \mathrm{~nm} \pm 0.4 \mathrm{~nm}$ have been chosen from the literature data [10]. This wavelength was chosen because at this wavelength maximum of reflectance exists [10] [11].

\section{Results and Discussion}

The result of measuring reflectance at $680 \mathrm{~nm}$ for several age groups was presented in Figure 2 and Figure 3. Figure 2 presents a change of normalized reflectance of a fingertip for nonsmoker upon the age of the subject. One can see the nonlinear reflectance change with the maximum at about $31-32$ year after that slowly decrease. As one connects the reflectance with hemoglobin concentration (higher concentration smaller reflectance greater absorption and vice versa) one can conclude that maximum reflectance is a direct consequence of the minimum presence of hemoglobin in the skin. Dependence of other important optical properties of skin, stimulated weak emission, upon age was observed [8]. Appearing maximum make that all reasonable explanations based on assumption that the significant changes in skin physiology and pathology due the year and, therefore in a change in their optical properties become unacceptable. Therefore, 


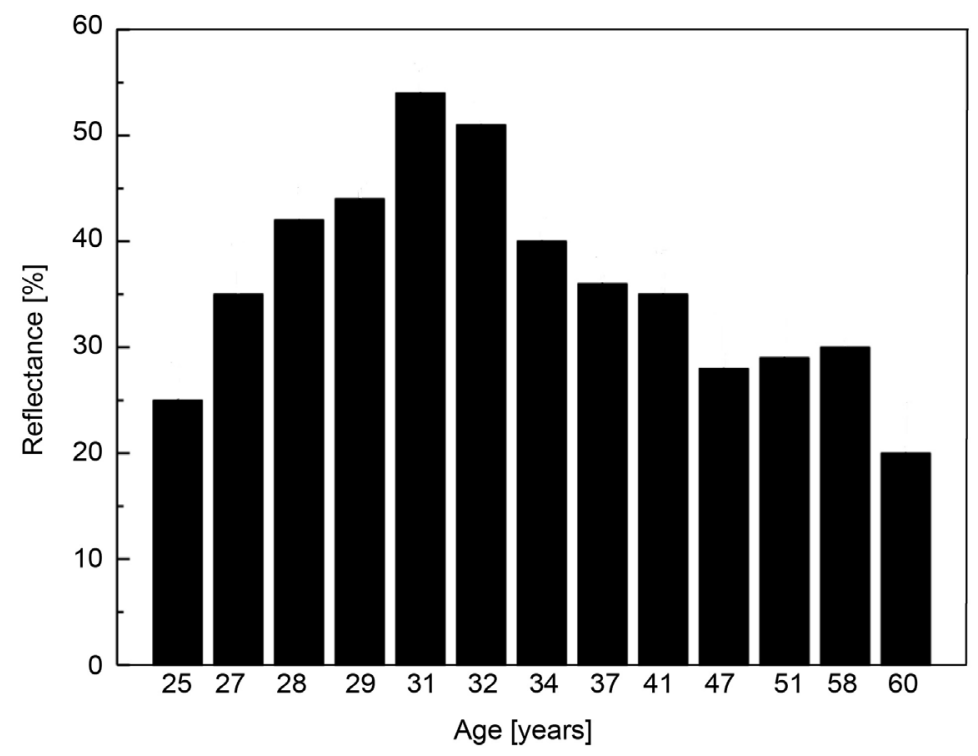

Figure 2. Dependence of the normalized reflectance (\%) the human fingerprints upon age.

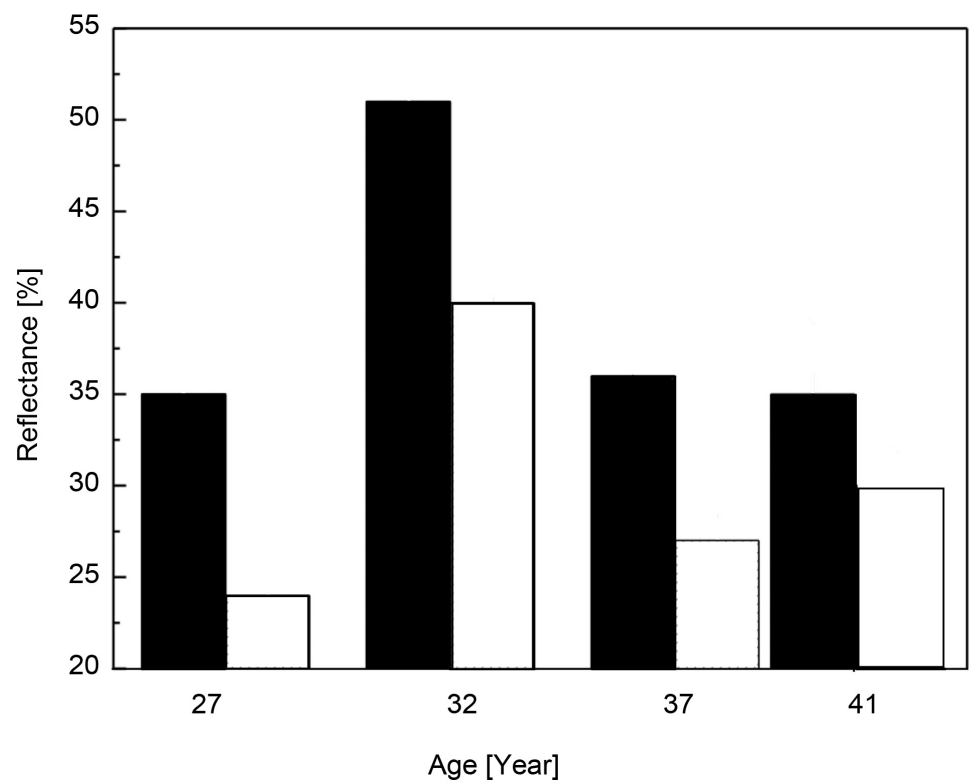

Figure 3. Reflectance (\%) from smoker (•) and nonsmoker ( $\square$ ) fingertip.

this will be the object of detailed further in research.

Ultra weak bioluminescence from a human fingertip, performed on a limited number of objects, shows a connection with smoking [8]. But due to the small number of experiments, a clear connection between bioluminescence and smoking was not observed. On the other hand, the effect of smoking was observed by measuring the ultra-weak light emission intensity of human blood [12]. Great confusion arises because besides smoking circumstance other unfavorable factors can cause a change in the optical properties of tissues or organs. A significant difference between the intensity of fluorescence for normal and arterioscle- 
rosis artery was found [13]. Also, laser-induced fluorescence shows a significant difference between human teeth with and without caries [14] and the difference between reflectance for cancerous and normal skin [15]. The obtained difference in reflection of the fingertip skin with the age of humans or in other words with the period of smoking is in agreement with literature data [16]. The smoking dose which is connecting with a period of consummations of cigarettes is related to skin pigment concentrations [17]. It is well known that the direct consequence of smoking is appearing reduction in arteries diameter [18]. Reducing the diameter of the arteries causes a decrease in the amount of blood that passes through the arteries and thus a decrease in the hemoglobin concentration. Smaller arteries diameter can cause smaller blood flow and at this way smaller hemoglobin concentration. Therefore, a possible explanation for a significant increase in reflectance for smokers may be an insignificant decrease in the concentration of the hemoglobin concentration, which maximum absorbed at 680 $\mathrm{nm}$, due to the decrease in arteries' diameter induced by nicotine [18]. Or in the simple relation: skin $\rightarrow$ smaller concentration of skin pigments $\rightarrow$ higher reflectance.

Taking the mentioned above into account, in our opinion, one reasonable explanation for the obtained change of the reflectance for the index finger (skin of index finger) could lie in the fact that the prolongation of the age of smoking increases the concentration of pigment in the skin and therefore the reflection. Or in the simple relation: skin $\rightarrow$ smaller concentration of skin pigments $\rightarrow$ higher reflectance.

Figure 3 compares the normalized fingerprint between smokers and no smokers. Analyzing Figure 3, one can see that a significant difference between the reflectance of smokers and nonsmokers exists. Exactly, the difference was between $23 \%$ and $100 \%$. The maximum difference appears in the case (27 years old participants) where index finger reflectance for the smokers is about four times greater than for nonsmokers. The obtained great increase in reflectance may be understood as a dramatic response of the young human body on unfavorable factors in order to keep steady-state conditions in the organism. There are several examples in the literature where the effect of smoking on skin and tissue optical properties was observed.

Chemicals in cigarette smoke cause the blood to thicken and form clots inside veins and arteries. Some experiments have shown that that cigarette smoke had an oxidative effect on the surface of the human skin [19]. Also, in literature data, one can find that smoking dramatically changes pigments which give the color to the skin exactly to the hemoglobin [20] and melanin [21]. On the other hand, some experiments have shown that cigarette smoking cause irregular pigmentation, and dryness of the skin [22] [23] [24]. Taking into account the mentioned above, a possible explanation in differences in reflections of fingertips between smoker and nonsmoker can be explained with a change in pigment concentration and skin quality. Also, with aging, the organism loses the ability to generi- 
cally defend itself from adverse effects, in this case, tobacco smoke.

In agreement with the mentioned above, it is clear that in order to make our research more precise and to obtain a real correlation between index finger reflectance and changing in arteries diameter, we must perform experiments using an electron microscope. This is the object of our further experiments.

\section{Conclusion}

Presented results lead to the conclusion that a direct connection between the physiological and pathological condition of human skin from a fingertip and the reflectance exists, and is moderated by age and smoking circumstance. Also, measuring reflectance from fingertips can give useful information about a possible change in the physiological and pathological condition of the human body, skin, caused by different unfavorable factors. The novelty of the study is reflected in the new insights into investigating the physiological and pathological condition of human body by considering the optical properties of the index finger skin.

\section{Conflicts of Interest}

The authors declare no conflicts of interest regarding the publication of this paper.

\section{References}

[1] Lee, H., Ko, H. and Lee, J. (2016) Reflectance Pulse Oximetry: Practical Issues and Limitations. ICT Express, 2, 195-198. https://doi.org/10.1016/j.icte.2016.10.004

[2] Njoum, H. and Kyriacou, P.A. (2013) Investigation of Finger Reflectance Photoplethysmography in Volunteers Undergoing a Local Sympathetic Stimulation. Journal of Physics: Conference Series, 450, 012012-012018. https://doi.org/10.1088/1742-6596/450/1/012012

[3] Cooksey, C.C., Tsai, B.K. and Allen, D.W. (2015) Spectral Reflectance Variability of Skin and Attributing Factors. Proceedings of SPIE Defense and Security Symposium, 9461. https://doi.org/10.1117/12.2184485

[4] Erdmann, W. and Bruley, D.F. (1992) Oxigen Transport in Tissue XIV. Springer Science+Businis Media LLC, New York.

[5] Bardou, F., Bouchaud, P.J., Aspect, A. and Cohen-Tannoudji, C. (2002) Lévy Statistics and Laser Cooling, Cambridge, Cambridge University Press, Cambridge. https://doi.org/10.1017/CBO9780511755668

[6] Imhof, R.E., Birch, D.J.S., Thornley, F.R., Gilchrist, J.R. and Strivens, T.A. (1990) Optothermal Transient Emission Radiometry. Physics in Medicine \& Biology, 35, 95-102.

[7] Konig, K., Ruck, A. and Schneckenburger, H. (1992) Fluorescence Detection and Photodynamic Activity of Endogenous Protoporphyrin in Human Skin. Optical Engineering, 31, 1470-1474. https://doi.org/10.1117/12.57700

[8] Zheng, R. (1988) On the Integrated Biophysical States of the Human Body Based on Ultra-Weak Bioluminescence of Fingertips. Journal of Luminescence, 40-41, 825-826. https://doi.org/10.1016/0022-2313(88)90455-3 
[9] Alfano, A.R., Tang, G., Pradhan, A., Lam, W., Choy, D. and Opher, E. (1987) Fluorescence Spectra from Cancerous and Normal Human Breast and Lung Tissues. IEEE Journal of Quantum Electronics, 23, 1806-1811. https://doi.org/10.1109/JQE.1987.1073234

[10] Jacquez, J.A., Huss, J., Mckeehan, W., Dimitroff, J.M. and Kuppenheim, H.F. (1955) Spectral Reflectance of Human Skin in the Region 0.7-2.6 $\mu$. Journal of Applied Physiology, 8, 297-299. https://doi.org/10.1152/jappl.1955.8.3.297

[11] Eckoldt, K. (1961) Die optischen Eigenscaften der menschlichen Haut in IR-Bereich. In: Christensen, B.C., Ed., Progress in Photobiology, Elsevier, Amsterdam, 489.

[12] Inaba, H. (1988) Super-High Sensitivity Systems for Detection and Spectral Analysis of Ultraweak Photon Emission from Biological Cell Cells and Tissues. Experientia, 44, 550-559. https://doi.org/10.1007/BF01953302

[13] Kittrell, C., Willett, R.L., De Los Santos-Pacheo, C., Ratliff, N.B., Kramer, J.R., Malk, E.G. and Feld, M.S. (1985) Diagnosis of Fibrous Arterial Atherosclerosis Using Fluorescence. Applied Optics, 24, 2280-2281. https://doi.org/10.1364/AO.24.002280

[14] Alfano, R.R., Tata, D.D., Cordero, J., Tomashefsky, P., Longo, F.W. and Alfano, M.A. (1984) Laser Induced Fluorescence Spectroscopy from Native Cancerous and Normal Tissue. IEEE Journal of Quantum Electronics, QE-20, 1507-1501. https://doi.org/10.1109/JQE.1984.1072322

[15] Ulrich, M., Lange-Asschenfeldt, S. and González, S. (2012) In Vivo Reflectance Confocal Microscopy for Early Diagnosis of Nonmelanoma Skin Cancer. Actas Dermo-Sifiliográficas, 103, 784-789. https://doi.org/10.1016/j.ad.2011.10.017

[16] Hedin, C.A., Pindborg, J.J. and Axell, T. (1993) Disappearance of Smoker's Melanosis after Reducing Smoking. Journal of Oral Pathology \& Medicine, 22, 228-230. https://doi.org/10.1111/j.1600-0714.1993.tb01061.x

[17] Araki, S., Murata, K., Ushio, K. and Sakai, R. (1983) Dose-Response Relationship between Tobacco Consumption and Melanin Pigmentation in the Attached Gingiva. Archives of Environmental Health, 38, 375-378. https://doi.org/10.1080/00039896.1983.10545823

[18] Walter, H. and Herbertm, D. (1975) Cigarette Smoking and Arteriographically Demonstrable Coronary Artery Disease. Clinical Investigations, 67, 49-62. https://doi.org/10.1378/chest.67.1.49

[19] Egawa, M., Kohno, Y. and Kumano, Y. (1999) Oxidative Effects of Cigarette Smoke on the Human Skin. International Journal of Cosmetic Science, 21, 83-98. https://doi.org/10.1046/j.1467-2494.1999.181656.x

[20] Nordenberg, D., Yip, R. and Binkin, N.J. (1990) The Effect of Cigarette Smoking on Hemoglobin Levels and Anemia Screening. JAMA, 264, 1556-1559.

https://doi.org/10.1001/jama.264.12.1556

[21] Araki, S., Murata, K., Ushio, K. and Sakai, R. (1983) Dose-Response Relationship between Tobacco Consumption and Melanin Pigmentation in the Attached Gingiva. Archives of Environmental Health, 38, 375-378. https://doi.org/10.1080/00039896.1983.10545823

[22] Green, A., Beardmore, G., Hart, V., Leslie, D., Marks, R. and Staines, D. (1988) Skin Cancer in a Queensland Population. Journal of the American Academy of Dermatology, 19, 1045-1052. https://doi.org/10.1016/S0190-9622(88)70270-4

[23] Kricker, A., Armstrong, B.K., English, D.R. and Heenan, P.J. (1991) Pigmentary and Cutaneous Risk Factors for Non-Melanocytic Skin Cancer-A Case-Control Study. International Journal of Cancer, 48, 650-662. https://doi.org/10.1002/ijc.2910480504 
[24] Kennedy, C., Bastiaens, M.T., Willemze, R., Bouwes Bavinck, J.N. and Bajdik, C.D. (2003) Effect of Smoking and Sun on the Aging Skin. Journal of Investigative Dermatology, 120, 548-554. https://doi.org/10.1046/j.1523-1747.2003.12092.x 